\title{
Identification of novel Bruton's tyrosine kinase mutations in 10 unrelated subjects with $\mathrm{X}$ linked agammaglobulinaemia
}

Rik A Brooimans, Adriënne J A M van den Berg, Ger T Rijkers, Lieke A M Sanders, Johannes K Ploos van Amstel, Marcel G J Tilanus, Marina J A L Grubben, Ben J M Zegers

Department of Immunology, University Children's Hospital "Het Wilhelmina Kinderziekenhuis", PO Box 18009, 3501 CA Utrecht, The

Netherlands

R A Brooimans

A J A $M$ van den Berg

G T Rijkers

L A M Sanders

B J M Zegers

DNA Laboratory, Clinical Genetics

Centre Utrecht, PO

Box 18009, 3501 CA

Utrecht, The

Netherlands

J K Ploos van Amstel

Department of Pathology, University Hospital Utrecht, PO

Box 85500, 3508 GA

Utrecht, The

Netherlands

M G J Tilanus

Department of

General Internal

Medicine, Nijmegen

University Hospital,

PO Box 9101, 6500 HB

Nijmegen, The

Netherlands

M J A L Grubben

Correspondence to:

Dr Brooimans.

Received 17 September 1996 Revised version accepted for publication 30 January 1997

\begin{abstract}
Mutations of the Bruton's tyrosine kinase (Btk) gene cause $X$ linked agammaglobulinaemia (XLA). This inherited immunodeficiency disease causes an arrest in $B$ cell differentiation of pre-B cells to mature $B$ cells. In this study we report the characterisation of mutations in the Btk gene in 10 unrelated XLA families. The screening approach we used was based on reverse transcriptase PCR and direct cycle sequencing of the amplified products followed by analysis of the observed mutations at the level of genomic DNA. The single strand confirmation polymorphism (SSCP) technique was used for assessment of the carriers in some of these families. Various mutations throughout the coding gene were observed, including missense and nonsense mutations, a deletion, and several splicing defects. None of the mutations except one has been previously described. There were three point mutations resulting in a single amino acid substitution. One of these missense mutations was observed in a conserved region of the PH domain, the other two were found in the src homology domain 2 that is involved in phosphotyrosyl peptide binding. Two mutations were single base pair substitutions resulting in premature stop codons. In four patients abnormal Btk transcripts were found that were the result of aberrant splicing. One small deletion was observed causing a frameshift and a secondary premature termination signal. Characterisation of the mutations responsible for XLA allowed us to diagnose the disease conclusively and identify the phenotypically normal female carriers. (F Med Genet 1997;34:484-488)
\end{abstract}

Keywords: X linked agammaglobulinaemia; Bruton's tyrosine kinase; immunodeficiency

$\mathrm{X}$ linked agammaglobulinaemia (XLA) was the first disorder of humoral immunity to be described as early as 1952 by Bruton. ${ }^{1}$ This inherited immunodeficiency is characterised by protracted and recurrent bacterial infections. Affected males have severely decreased numbers of circulating mature B lymphocytes and show a lack of all immunoglobulin (Ig) isotypes. ${ }^{2}$ In the bone marrow the number of $B$ lymphocyte precursors, identified by Ig heavy chain expression in the cytoplasm, are normal or decreased. Therefore, XLA reflects an arrest in differentiation of pre-B cells to later $B$ cell stages. ${ }^{3}$ The cellular defect is limited to the B cell line, since female XLA carriers manifest a unilateral $\mathrm{X}$ chromosome inactivation in the peripheral blood B cell population. ${ }^{4}$ The disorder is usually diagnosed quite early in life and treatment consists of a lifetime of regular Ig infusions. In 1993 the causative gene in XLA was identified as a cytoplasmatic protein tyrosine kinase, named Bruton's tyrosine kinase (Btk). ${ }^{56}$ This gene has been mapped to $\mathrm{Xq} 21.3-\mathrm{Xq} 22^{7}$ and is widely expressed throughout haematopoietic cell lines, including nearly all stages of B cell development, but is found at negligible levels in $T$ cells and is downregulated in plasma cells. ${ }^{8}$ The $37.5 \mathrm{~kb}$ Btk locus contains 19 exons, 18 of which code for a $77 \mathrm{kDa}$ protein. ${ }^{9}$

Btk belongs to a subfamily of Src related cytoplasmatic protein tyrosine kinases (PTKs), including Tec II ${ }^{10} \mathrm{Bmx},{ }^{11} \mathrm{Txk},{ }^{12} \mathrm{Itk},{ }^{13}$ and Dsrc $28 \mathrm{C},{ }^{14}$ also known as the Tec family. Proteins of the Tec family consist of five distinct structural domains, a unique $\mathrm{N}$-terminal region, which is comprised of a pleckstrin homology ( $\mathrm{PH})$ domain ${ }^{15}$ and a Tec homology (TH) domain, ${ }^{16}$ followed by $\mathrm{Src}$ homology ( $\mathrm{SH}) 3$, $\mathrm{SH} 2$, and catalytic (SH1) domains. $\mathrm{PH}$ domains have been found in a number of proteins involved in intracellular signalling pathways. Protein kinase $\mathrm{C}$ and heterotrimeric $\mathrm{G}$ protein have been proposed to bind to the Btk $\mathrm{PH}$ domain. ${ }^{17}{ }^{18}$ The $\mathrm{TH}$ domain contains two proline rich regions which have been shown to interact with the $\mathrm{SH} 3$ domains of Src protein tyrosine kinases Fyn, Lyn, and Hck. ${ }^{19}$ Analysis of Src family tyrosine kinases suggests that $\mathrm{SH} 2$ and $\mathrm{SH} 3$ domains are involved in intermolecular recognition and the formation of heterodimeric protein complexes. SH2 domains directly recognise phosphorylated tyrosine residues and the $\mathrm{SH} 3$ domain is implicated in the regulation of protein-protein interactions via recognition of specific proline rich peptide sequences. ${ }^{20}$ Signal transduction pathways are likely to be controlled by the formation of these protein complexes. ${ }^{21}$

In general, the study of null mutations in a gene that results in an inherited disorder will provide information about the functionally important domains of the gene product. In the 
Table 1 Oligonucleotide primers for the amplification of the $c D N A$ of Btk

\begin{tabular}{lll}
\hline Primer & Sequence & Position $^{*}$ \\
\hline BTK A F & GTGAACTCCAGAAAGAAGAAGCT & 110 \\
BTK A R & TCCTGTTCTCCAAAATTTGGCAG & 646 \\
BTK B F & CAGCCAAAAATGCTATGGGCTG & 605 \\
BTK B R & AATGACGTATCACCCCTTGAGG & 1132 \\
BTK C F & GTTTGCTAAATCCACAGGGGAC & 1089 \\
BTK C R & CATCCTTGCACATCTCTAGCAG & 1645 \\
BTK D F & TCCAGACTCAGCAGCTGCTAG & 1610 \\
BTK D R & ACCAAGAAGCTTATTGGCGAGC & 2134
\end{tabular}

«The positions of the 5 ' nucleotide of the primers are derived from the human Btk cDNA. ${ }^{5}$

case of Btk it could indicate clues about its role in $B$ cell differentiation. Furthermore, mutation analysis is more accurate in diagnosing affected subjects and carriers, especially in those cases which show a milder or later onset of a humoral immunodeficiency in the absence of a family history of $\mathrm{X}$ linked inheritance. Mutations in the Btk gene have now been identified by several groups in about 200 unrelated XLA patients. Mutations reported to date include deletions, insertions, and missense or nonsense mutations and have been found in every domain of Btk. ${ }^{22} 23$ The first mutation data that were reported suggested that the severity of the XLA phenotype is dependent upon the site and nature of the mutation. ${ }^{924} 25$ Now that data on so many XLA patients have been reported, it has become evident that there is no strict correlation between the site of the mutation and the severity of the clinical and immunological expression of the disease, implying that other factors might determine the phenotype as well.

This study was undertaken to analyse 14 subjects from 10 unrelated families who represent either familial or sporadic cases of XLA, based on a clinical and immunological diagnosis. Here we report the characterisation of mutations found in the Btk gene of these subjects.

\section{Materials and methods \\ PATIENTS}

Fourteen Dutch patients from 10 unrelated families were included in the study. All patients were male, aged between 2 and 38 years, and diagnosed as having XLA. The diagnosis of XLA was based on the absence or very low levels of B cells in the circulation, low levels of immunoglobulins, and a history of recurrent bacterial infections.

RNA AND DNA ISOLATION

PBMCs were prepared from fresh blood by Ficoll (Pharmacia) separation. RNA was extracted from the PBMC fraction with RNAzol $\mathrm{B}$ (Cinna/Biotex) and used for first strand cDNA synthesis with AMV reverse transcriptase and $\operatorname{Not} \mathrm{I}-\mathrm{d}(\mathrm{T})_{18}$ primer. Genomic DNA was purified with a QIAgen isolation kit according to the manufacturer's instructions.

\section{PCR AMPLIFICATION}

PCR amplification was carried out in four overlapping sections using the oligonucleotide primer sets listed in table 1 . PCR reactions were performed in a total volume of $100 \mu \mathrm{l}$, consisting of $3 \mu \mathrm{l} \mathrm{cDNA}, 50 \mathrm{mmol} / 1 \mathrm{KCl}, 10$ $\mathrm{mmol} / 1$ Tris- $\mathrm{HCl}, \mathrm{pH} 8.4,2 \mathrm{mmol} / 1 \mathrm{MgCl}_{2}, 50$ $\mathrm{pmol}$ of each primer, $0.4 \mathrm{mmol} / 1 \mathrm{dNTPs}$, and 1 U AmpliTaq (Perkin-Elmer). Amplification was for 35 cycles with denaturation at $94^{\circ} \mathrm{C}$, annealing at $60^{\circ} \mathrm{C}$, and extension at $72^{\circ} \mathrm{C}$, each for 30 seconds on a Perkin-Elmer Thermal Cycler system 9600.

Genomic DNA PCR amplification was carried out with the sets of primers flanking all exons as reported by Vorechovsky et al, ${ }^{26}$ using their cycling protocol with some modifications.

\section{SEQUENCE ANALYSIS}

Preamplified PCR products were used as templates for direct cycle sequence kit (PerkinElmer) according to the manufacturer's instructions.

\section{PCR-SSCP}

PCR products for single strand conformation polymorphism (SSCP) were generated according the same protocol as described above. The detection of SSCPs was carried out on non-denaturating agarose gels on the PhastSystem TM (Pharmacia,Sweden). After dilution $1: 1$ in formamide loading buffer, the amplification products were loaded onto $20 \%$ homogeneous phastgels (Pharmacia). Electrophoresis was done at $15^{\circ} \mathrm{C}$ at $250 \mathrm{Vh}$. DNA was visualised by silver staining according to the instructions of the manufacturer.

\section{Results}

MUTATION ANALYSIS OF THE BTK GENE

Characterisation of mutations in the Btk gene of XLA patients was carried out by using the following strategy. To identify mutations in the coding region of Btk, cDNA was prepared by a reverse transcriptase $P C R$ reaction from peripheral blood mononuclear cells. The amplified products encompassed the coding sequence in four overlapping segments. The PCR product of each segment was directly sequenced by cycle sequencing. To analyse and confirm the identified mutations in patient cDNA, we amplified and sequenced patient genomic DNA with primers flanking the exon involved. Mutation analysis was performed in a total of 14 subjects from 10 unrelated families of Dutch origin. Patients were diagnosed as classical XLA based upon the number of peripheral B cells and serum Ig levels. In four families there were two male members affected with $\mathrm{X}$ linked immunodeficiency disease; the other patients were sporadic cases. All patients analysed showed alterations in the Btk gene, which are summarised in table 2. Various mutations were observed, including insertions, deletions, and missense or nonsense mutations. Five patients manifested single point mutations in the coding sequence. Patient 6 showed a $\mathrm{T} \rightarrow \mathrm{C}$ change at position 164 replacing the hydrophobic non-polar amino acid leucine with another hydrophobic non-polar amino acid proline, at residue 11 in the $\mathrm{PH}$ domain. The Leu 11 amino acid is strongly conserved in residues that form the first $\beta$ sheet of known $\mathrm{PH}$ domain sequences, like pleckstrin, spectrin, and dynamin. ${ }^{27}$ In patient 4 , a $T \rightarrow G$ substitution was found at position 1218 that 
Table 2 Mutations of the Btk gene in 10 unrelated XLA patients

\begin{tabular}{llllll}
\hline Patient & $\begin{array}{l}\text { Affected } \\
\text { relative }\end{array}$ & Nucleotide change & $\begin{array}{l}\text { Alteration of coding } \\
\text { sequence }\end{array}$ & $\begin{array}{l}\text { Btk } \\
\text { domain }\end{array}$ & Genomic DNA mutation \\
\hline 6 & + & $164 \mathrm{~T} \rightarrow \mathrm{C}$ & Leu1 1 Pro & $\mathrm{PH}$ & Exon 2 \\
2 & + & $773-908$ & Aberrant splicing & SH3 & $+1 \mathrm{nt}$ intron $8 \mathrm{~g}>\mathrm{t}$ \\
7 & - & $895 \mathrm{C} \rightarrow \mathrm{T}$ & Arg255stop & SH3 & Exon 8 \\
3 & - & $935-936$ & Frameshift & SH2 & Exon 9 \\
8 & - & $1114 \mathrm{C} \rightarrow \mathrm{T}$ & Gln308stop & SH2 & Exon 12 \\
4 & - & $1218 \mathrm{~T} \rightarrow \mathrm{G}$ & His362Gln & SH2 & Exon 12 \\
9 & - & $1225 \mathrm{~A} \rightarrow \mathrm{T}$ & Asn365Tyr & Kinase & Exon 12 \\
10 & + & $\star 20 \mathrm{nt}$ at 1309 & Aberrant splicing & Kinase & del +2 nt intron 13 \\
5 & + & $1310-1481$ & Aberrant splicing & Kinase & +1 nt intron $14 \mathrm{~g}>\mathrm{t}$ \\
11 & - & $\star 90 \mathrm{nt}$ at 1481 & Aberrant splicing & Kinase & $+5 \mathrm{nt}$ intron $14 \mathrm{~g}>\mathrm{a}$ \\
\hline
\end{tabular}

altered residue 362 in the $\mathrm{SH} 2$ domain from a basic histidine to an uncharged glutamine. Based on the crystal structure of the $\mathrm{SH} 2$ domain of $\mathrm{v}$-src, this mutation leads to a disturbed phosphotyrosyl peptide binding. ${ }^{28}$ Patient 9 also showed a missense mutation in the $\mathrm{SH} 2$ domain changing nucleotide 1225 from A to T causing substitution of residue 365

A
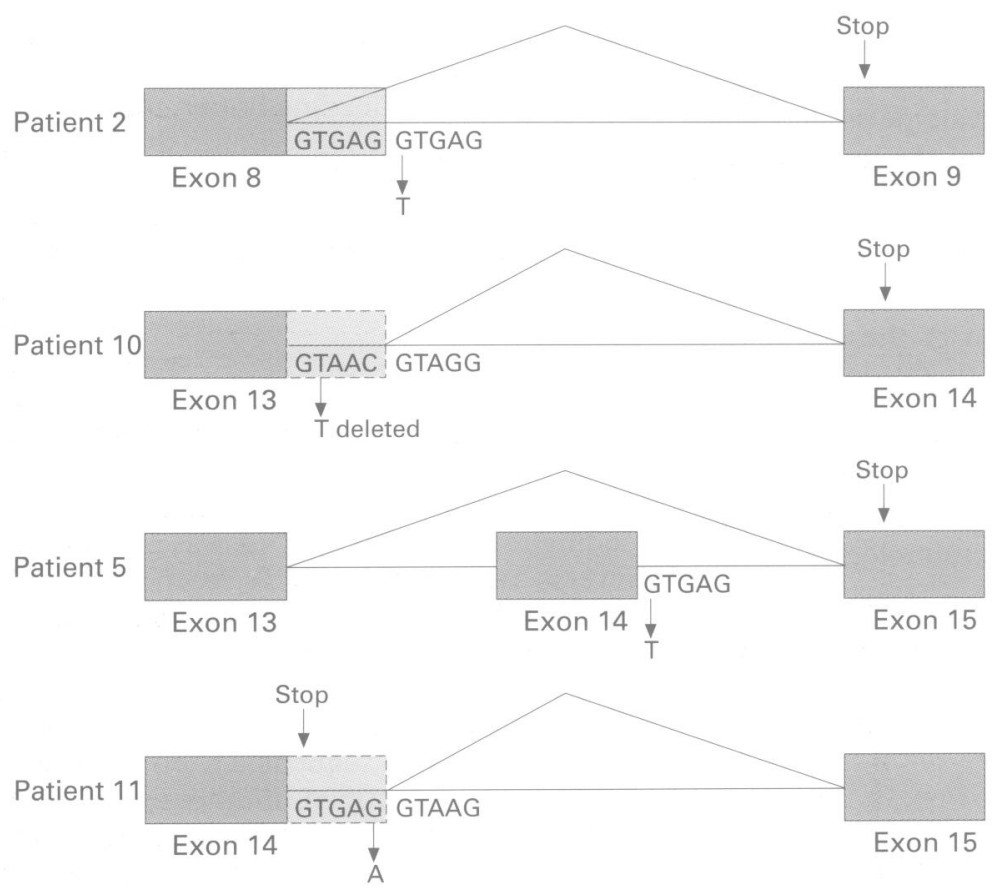

B

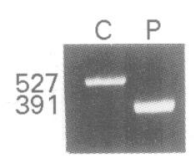

C

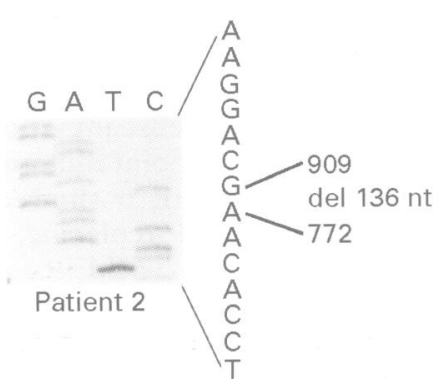

Figure 1 Mutation analysis of aberrant splicing in the Btk gene of XLA subjects. $(A)$ Schematic representation illustrating the aberrant splicing products in four XLA patients. (B) Size analysis of amplified cDNA product of patient $2(P)$ and a normal control. $(C)$ Sequence analysis showing the deletion in the transcript of patient 2 . (D) Identification of a point mutation in the splice donor site of the patient's genomic DNA. from an amino acid asparagine for an aromatic tyrosine. This residue seems not to be directly involved in phosphotyrosyl peptide binding, but the effect of the mutation might be structural. In patient 7 , a mutation $\mathrm{C} \rightarrow \mathrm{T}$ at position 895 was identified which results in a premature termination signal at amino acid residue 255. This mutation has also been described in two other unrelated XLA patients. ${ }^{29}{ }^{30}$ Patient 8 had a $\mathrm{C} \rightarrow \mathrm{T}$ nonsense mutation at position 1114 , giving rise to a premature stop codon at amino acid residue 308 in the $\mathrm{SH} 2$ domain of Btk.

In four patients, abnormal sized Btk transcripts were found, which were the result of aberrant splicing. Fig 1A shows a schematic representation of the effects of splicing mutations in these patients. The larger deletions and insertions had already been observed by size analysis of the four cDNA segments in comparison with normal sized PCR products. The PCR product obtained with primers Btk B $\mathrm{F}$ and Btk B R was smaller in patient $2(391 \mathrm{bp})$ than the control (527 bp) (fig 1B). Sequence analysis of the smaller fragment showed that the transcript lacked nucleotides 773 to 908 (fig 1C). According to the location of the deletion or insertion in the transcript, the appropriate exon-intron boundaries were sequenced using genomic DNA from the patient as template. The observed deletion in patient 2 is compatible with a cryptic donor splice site present within exon 8 , indicating the loss of the donor splice site located downstream of the gene. Indeed, as shown in fig $1 \mathrm{D}$, genomic sequence analysis showed a point mutation $\mathrm{G} \rightarrow \mathrm{T}$ involving the first nucleotide of intron 8 . The resulting frameshift introduced a premature termination signal 18 amino acids downstream of codon 213 . In patient 10 , a deletion of a $T$ at the +2 position of the donor splice site in intron 13 activated a cryptic splice 21 nucleotides downstream, which resulted in an insertion of 20 bases encoded by intron 13 in the Btk transcript. The resulting frameshift introduced a termination signal 17 amino acids downstream of codon 392. In the Btk transcript of patient 5 , a deletion of 172 bases ( $\mathrm{nt}$ 1310-1481) was observed. The deleted sequence is encoded by exon 14, indicating an aberrant splicing mechanism. Sequence analysis of the genomic template of this patient showed a nucleotide substitution $G \rightarrow T$ at the +1 position of the donor splice junction in intron 14. The resulting frameshift introduced a stop codon 34 residues downstream of codon 392. Patient 11 showed a $G \rightarrow A$ change at position +5 of intron 15 , activating a cryptic donor splice site further downstream. This resulted in the addition of 90 bases of intron 15 to the Btk message giving rise to an immediate termination signal following codon 449. Patient 3 manifested a deletion of two bases beginning at nucleotide position 934 or 935 , which would result in a premature stop codon 3 amino acids downstream of codon 267 .

CARRIER DETECTION WITH SSCP

With the exact mutation characterised for each XLA patient, we could now determine which 


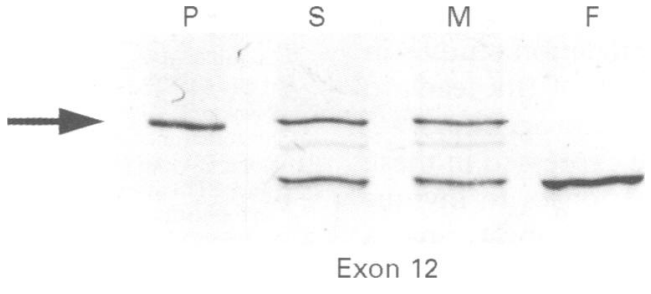

Figure 2 SSCP analysis of exon 12 of the Btk gene of proband $6(P)$ and both his parents ( $F=$ father, $M=$ mother) and sister (S). The arrow indicates the mobility shift caused by the polymorphism. Gels were run on a Phastsystem and silver stained.

other relatives had inherited the same defective gene. This was done by using SSCP analysis with primer combinations flanking the exons involved. ${ }^{26}$ SSCP analysis of exon 12 from the relatives of patient 4 , shown in fig 2 , indicated that the mother of the affected boy and his sister were carriers. SSCP analysis was also performed for screening of the carrier status of female relatives of patients in family $2,3,6$, and 10 (data not shown).

\section{Discussion}

In this study we have identified the mutations in the Btk gene of 14 patients from 10 unrelated families with a classical XLA phenotype. Mutation analysis performed in all patients, who were selected on the basis of recurrent infections or $B$ cell levels of less than $1 \%$, identified a mutation in the coding region of the Btk gene. The identified mutations were submitted to the Btk mutation database that has been established by Vihinen et al. ${ }^{31}$ All but one of these mutations differed from previously reported mutations in the BTK gene and are all spread throughout the gene. The mutation analysis approach that was chosen was based upon first screening patients' cDNA and confirming the detected mutation on genomic DNA templates, especially to identify those mutations in which aberrant splicing is involved. Others have shown that PCR-SSCP analysis is useful in screening for new mutations in XLA patients. ${ }^{25}{ }^{26}{ }^{32}$ However, because of the genomic organisation of 19 exons which encompasses the entire Btk gene, one has to use 20 to 25 primer pairs for this analysis. From a cost effectiveness point of view we decided to use the PCR-SSCP analysis only for screening of carriers.

To date, in more than 200 unrelated XLA patients, mutations scattered all along the Btk gene have been identified. ${ }^{22}{ }^{23}$ In this study three patients out of a total of 14 had a missense mutation. Patient 6 had a single amino acid substitution that is a conserved residue of the first $\beta$ sheet of the $\mathrm{PH}$ domain. Several other missense mutations have been described located in this PH domain. Based on the three dimensional structure of the Btk PH domain these mutated residues could form a binding site, since most of them were located close to each other on the surface of the molecule, suggesting that this domain is involved in protein-protein interactions. ${ }^{27}{ }^{33}$ For instance, Btk PH domains have been shown to interact with protein kinase C. ${ }^{17}$ The other two observed missense mutations were localised in the $\mathrm{SH} 2$ domain of Btk. Alignment between this domain and $\mathrm{SH} 2$ of $\mathrm{v}-\mathrm{Src}$ indicated that the mutated residue 362 is directly involved in binding to phosphotyrosyl peptides. ${ }^{28}$

Most of the other identified mutations caused rearranged Btk transcripts or premature termination signals or both, encoding at best a truncated protein lacking the complete or functional part of the kinase domain. All of these patients showed the typical XLA phenotype with recurrent infections, B cell numbers less than $1 \%$, and low serum Ig levels. The splice mutation detected in XLA patient 11 did not occur in the absolutely conserved position of the donor splice junction, ${ }^{34}$ leaving the possibility that some normal Btk transcript is produced. On agarose gels no normal sized PCR product was detectable (data not shown). Furthermore it has been shown by others that several mutations at this position abolish normal splicing and result in similar rearrangements. ${ }^{35-37}$ From various reports it has become clear that it is very difficult to correlate the nature of a mutation with the severity of the disease. There have been mutations found causing premature stop codons and frameshifts affecting essential domains of Btk in patients with clinically mild phenotypes. ${ }^{25}$ Even between individual patients within one family with the same genotype, discordant phenotypes can occur. ${ }^{38}$ Furthermore, a recent study has shown that the majority of identified mutations, including missense mutations, lead to no detectable protein kinase activity. ${ }^{39}$ This suggests that these mutations could have different effects on mRNA and protein stability.

XLA carriers cannot be distinguished phenotypically from normal subjects. However, by SSCP analysis it is possible to detect an XLA carrier by determining the presence of a normal band and an altered band identical to that of the patient. Four families were subjected to carrier analysis. Using PCR-SSCP we could successfully identify the carriers among family members (fig 2). This technique enables quick screening of relatives of the patient whether they have inherited the same defective gene or not and can also be used for prenatal diagnosis.

So far, a large amount of data have been obtained on mutation analysis of the Btk gene in typical XLA patients and screening methods have become more efficient for analysis of patients. It would be preferable to include more subjects with atypical disease in these Btk mutation analysis studies, such as, for instance, patients diagnosed as having common variable immunodeficiency diseases (CVID), since there are examples of an affected Btk gene in some of these patients. ${ }^{40}$

In general, one can conclude that three years after the identification of the causative gene responsible for XLA, the function of Btk in B cell differentiation still remains to be clarified. To date it is evident that there is no direct correlation between genotype and the clinical expression of the XLA phenotype, indicating that several other factors could play a role in the observed heterogeneity in XLA. For instance, genetic factors might influence the 
severity of the phenotype or past infections might play a role. Targeted deletion studies in mice have shown that the lack of Btk leads to impaired expression of $\mathrm{B}$ cell progenitors. ${ }^{41}{ }^{42}$ However, mature B cells are expressed in these mice, but show defective responses to thymus independent type II antigens, indicating a similar Xid phenotype to that observed in the CBA/N mice..$^{43}{ }^{44}$ It seems that the difficulty in explaining the observed heterogeneity differences in man and mice is correlated with the lack of knowledge concerning the functional role of the Btk protein. For a better understanding of the pathogenesis of XLA, it is therefore essential to study the expressed functional Btk protein, including determination of substrates of Btk in the B cell and the proteins that interact with the Btk gene product.

The authors would like to thank all the physicians who supplied us with patient samples, in particular Prof Dr J W M van der Meer (Department of General Internal Medicine, Nijmegen Meer (Department of General Internal Medicine, Nijmegen University Hospital, Nijmegen, The Netherlands), Dr E R de Graeff-Meeder, Dr N M Wulffraat, and W Kuis (Department of
Immunology, University Children's Hospital, Utrecht, The Netherlands).

1 Bruton OC. Agammaglobulinemia. Pediatrics 1952;9:722-8.

2 Conley ME. B cells in patients with X-linked agammaglobulinemia. F Immunol 1985;134:3070-4.

3 Pearl ER, Vogler LB, Okos AJ, et al. B lymphocyte precursors in human bone marrow: an analysis of normal individuals and patients with antibody deficiency states. $\mathscr{f}$ Immunol 1978;120:1169-75.

4 Conley ME, Brown P, Pickard AR, et al. Expression of the gene defect in X-linked agammaglobulinemia. $N$ Engl $f$ Med 1986;315:564-7.

5 Vetrie D, Vorechovsky I, Sideras P, et al. The gene involved in X-linked agammaglobulinaemia is a member of the src family of protein-tyrosine kinases. Nature 1993;361:226 33.

6 Tsukada S, Saffran DC, Rawlings DJ, et al. Deficient expression of a B cell cytoplasmic tyrosine kinase in human X-linked agammaglobulinemia. Cell 1993;72:279-90.

7 Kwan SP, Kunkel L, Bruns G, et al. Mapping of the X-linked agammaglobulinemia locus by use of restriction $\mathrm{X}$-linked agammaglobulinemia locus by use of restriction
fragment-length polymorphism. $\mathcal{F}$ Clin Invest $1986 ; 77: 649-$ 52 .

8 Smith CI, Baskin B, Humire Greiff P, et al. Expression of Bruton's agammaglobulinemia tyrosine kinase gene, BTK is selectively down-regulated in $\mathrm{T}$ lymphocytes and plasma cells. F Immunol 1994;152:557-65.

9 Ohta Y, Haire RN, Litman RT, et al. Genomic organization and structure of Bruton agammaglobulinemia tyrosine kinase: localization of mutations associated with varied clinical presentations and course in X chromosome-linked agammaglobulinemia. Proc Natl Acad Sci USA 1994;91 9062-6.

10 Mano H, Mano K, Tang B, et al. Expression of a novel form of Tec kinase in hematopoietic cells and mapping of the gene to chromosome 5 near Kit. Oncogene 1993;8:417-24.

11 Tamagnone L, Lahtinen I, Mustonen T, et al. BMX, a novel nonreceptor tyrosine kinase gene of the BTK/ITK/TEC/ TXK family located in chromosome Xp22.2. Oncogene 1994;9:3683-8.

12 Haire RN, Ohta Y, Lewis JE, et al. TXK, a novel human tyrosine kinase expressed in $\mathrm{T}$ cells shares sequence identity with Tec family kinases and maps to $4 \mathrm{p} 12$. Hum Mol Genet 1994;3:897-901.

13 Siliciano JD, Morrow TA, Desiderio SV. itk, a T cell specific tyrosine kinase gene inducible by interleukin 2. Proc Nat Acad Sci USA 1992;89:11194-8.

14 Gregory RJ, Kammermeyer KL, Vincent WS, Wadsworth SG. Primary sequence and developmental expression of a novel Drosophila melanogaster src gene. Moll Cell Bio 1987;7:2119-27.

15 Haslam RJ, Koide HB, Hemmings BA. Pleckstrin domain homology. Nature 1993;363:309-10.

16 Vihinen M, Nilsson L, Smith CI. Tec homology (TH) adjacent to the PH domain. FEBS Lett 1994;350:263-5.

17 Yao L, Kawakami Y, Kawakami T. The pleckstrin homology domain of Bruton tyrosine kinase interacts with protein kinase C. Proc Natl Acad Sci USA 1994;91:9175-9.

18 Tsukada S, Simon MI, Witte ON, Katz A. Binding of beta gamma subunits of heterotrimeric G proteins to the PH
domain of Bruton tyrosine kinase. Proc Natl Acad Sci USA 1994;91:11256-60.
19 Cheng G, Ye ZS, Baltimore D. Binding of Bruton's tyrosine kinase to Fyn, Lyn, or Hck through a Src homology 3 domain-mediated interaction. Proc Natl Acad Sci USA 1994;91:8152-5.

20 Pawson T, Gish GD. SH2 and SH3 domains: from structure to function. Cell 1992;71:359-62.

21 Koch CA, Anderson D, Moran MF, Ellis C, Pawson T. SH2 and $\mathrm{SH} 3$ domains: elements that control interactions of cytoplasmic signaling proteins. Science 1991;252:668-74.

22 Vihinen M, Cooper MD, De Saint Basile G, et al. BTKbase: a database of XLA-causing mutations. Immunol Today 1995;16:460-5

23 Vihinen M, Iwata T, Kinnon C, et al. BTKbase, mutation database for $\mathrm{X}$-linked agammaglobulinemia (XLA). Nucleic Acids Res 1996;24:160-5.

24 Saffran DC, Parolini O, Fitch Hilgenberg ME, et al. Brief report: a point mutation in the SH2 domain of Bruton's tyrosine kinase in atypical X-linked agammaglobulinemia. N Engl f Med 1994;330:1488-91.

25 Conley ME, Fitch Hilgenberg ME, Cleveland JL, Parolini $\mathrm{O}$, Rohrer J. Screening of genomic DNA to identify mutations in the gene for Bruton's tyrosine kinase. Hum Mol Genet 1994;3:1751-6.

26 Vorechovsky I, Vihinen M, De Saint Basile G, et al. DNA-based mutation analysis of Bruton's tyrosine kinase gene in patients with X-linked agammaglobulinaemia. gene in patients with X-linke
Hum Mol Genet 1995;4:51-8.

27 Vihinen M, Zvelebil MJJM, Zhu Q, et al. Structural basis for pleckstrin homology domain mutations in X-linked agammaglobulinemia. Biochemistry 1995;34:1475-81.

28 Waksman G, Shoelson SE, Pant N, Cowburn D, Kuriyan J. Binding of a high affinity phosphotyrosyl peptide to the Src SH2 domain: crystal structures of the complexed and peptide-free forms. Cell 1993;72:779-90

29 Bradley LA, Sweatman AK, Lovering RC, et al. Mutation detection in the X-linked agammaglobulinemia gene,
BTK, using single strand conformation polymorphism analysis. Hum Mol Genet 1994;3:79-83.

30 Jin $\mathrm{H}$, Webster $\mathrm{AD}$, Vihinen $\mathrm{M}$, et al. Identification of Btk mutations in 20 unrelated patients with X-linked agammamutations in 20 unrelated patients with X-linked agamm

31 Vihinen M, Brooimans RA, Kwan SP, et al. BTKBase: XLA-mutation registry. Immunol Today 1996;1 17:502-6.

32 Hagemann TL, Chen Y, Rosen FS, Kwan SP. Genomic organization of the Btk gene and exon scanning for mutations in patients with X-linked agammaglobulinemia. Hum Mol Genet 1994;3:1743-9.

33 de Weers M, Mensink RG, Kraakman ME, Schuurman RK Hendriks RW. Mutation analysis of the Bruton's tyrosine kinase gene in X-linked agammaglobulinemia: identification of a mutation which affects the same codon as is altered in immunodeficient xid mice. Hum Mol Genet 1994;3:161-6.

34 Frendeway D, Keller W. Stepwise assembly of a pre-mRNA splicing complex requires U-snRNPs and specific intron sequences. Cell 1985;42:355-67.

35 Duriez B, Duquesnoy P, Dastot F, et al. An exon-skipping mutation in the btk gene of a patient with X-linked agammaglobulinemia and isolated growth hormone deficiency. FEBS Lett 1994;346:165-70.

36 Lee B, Vitale E, Superti Furga A, Steinmann B, Ramirez F $G$ to $T$ transversion at position +5 of a splice donor site causes skipping of the preceding exon in the type III procollagen transcripts of a patient with Ehlers-Danlos syndrome type IV. $\mathcal{F}$ Biol Chem 1991;266:5256-9.

37 Ganguly A, Baldwin CT, Strobel D, et al. Heterozygous mutation in the $\mathrm{G}+5$ position of intron 33 of the pro-alpha 2(I) gene (COL1A2) that causes aberrant RNA splicing and gene (COL1A2) that causes aberrant RNA splicing and lethal osteogenesis imperfecta. Use of carbodiimide methods that decrease the extent of DNA sequencing necessary to define a
$1991 ; 266: 12035-40$.

38 Bykowsky MJ, Haire RN, Ohta $\mathrm{Y}$, et al. Discordant phenotype in siblings with $\mathrm{X}$-linked agammaglobulinemia. Am 7 Hum Genet 1996;58:477-83.

39 Hashimoto S, Tsukada S, Matsushita M, et al. Identification of Bruton's tyrosine kinase (Btk) gene mutations and characterization of the derived proteins in $35 \mathrm{X}$-linked agammaglobulinemia families: a nationwide study of Btk deficiency in Japan. Blood 1996;88:561-73.

40 Vorechkovsky I, Zhou JN, Vetrie D, et al. Molecular diagnosis of X-linked agammaglobulinaemia. Lancet 1993;341. 1153.

41 Khan WN, Alt FW, Gerstein RM, et al. Defective B cell development and function in Btk-deficient mice. Immunity 1995;3:283-99.

42 Kerner JD, Appleby MW, Mohr RN, et al. Impaired expansion of mouse B cell progenitors lacking Btk. Immunity 1995;3:301-12.

43 Thomas JD, Sideras P, Smith CI, et al. Colocalization of $\mathrm{X}$-linked agammaglobulinemia and X-linked immunodeficiency genes. Science 1993;261:355-8.

44 Rawlings DJ, Saffran DC, Tsukada S, et al. Mutation of unique region of Bruton's tyrosine kinase in immunodeficient XID mice. Science 1993;261:358-61. 